\title{
Health Monitoring Applications and the Transparency of Health
}

Bas de Boer*

\begin{abstract}
Mobile health (mHealth) is considered an important solution to the problem of delivering quality care in light of an aging population. One of the promises of mHealth is the development and promotion of mobile applications that support individuals in adopting a healthier lifestyle. In this paper, I will analyze in what sense health monitoring applications (HMAs) can establish a relationship between individuals and the 'healthiness' of their lifestyle. Through a phenomenological analysis of health, I show that our 'health' or the 'healthiness' of our lifestyle is transparent to us (eg, is not something that can be explicitly related to). As a consequence, mHealth is unable to make present 'health' or our 'healthy lifestyle' as a totality. I argue that this places limits on the extent to which mHealth can be understood as something that (dis)empowers citizens to take responsibility for their own health. Rather, so I suggest, HMAs give rise to a specific relation with one's physical or biological body, instead of making present one's health status. Therefore, discussions about HMAs should not be exclusively focus on how they (dis)empower citizens, but additionally focus on evaluating how our physical body is drawn out of its transparency.
\end{abstract}

\section{Introduction}

The European population is aging, making it likely that the number of people in need of medical treatment will grow rapidly in the coming decades. At the same time, healthcare systems face budgetary pressures already, and the situation is likely to worsen in light of the number of citizens that will be in need of care in the future. In light of this situation, the European Commission (EC) stated that ' $[\mathrm{H}]$ ealth systems need to shift from treatment to health promotion and disease prevention, from a focus on disease to a focus on well-being and individuals. [...] By using digital solutions such as wearables and mHealth apps, citizens can actively engage in health promotion and self-management of chronic conditions.' Accordingly, mobile health (mHealth) is considered

DOI: 10.21552/delphi/2019/3/6

* Bas de Boer, Postdoctoral Researcher, Philosophy Department, University of Twente. For correspondence: <s.o.m deboer@utwente.nl>

1 European Commission, 'On Enabling the Digital Transformation of Health and Care in the Digital Single Market; Empowering Citizens and Building a Healthier Society' COM (2018) 233 final

2 European Commission, 'Healthcare in Your Pocket: Unlocking the Potential of mHealth' Press Release (10 April 2014) 1 as an important solution to the problem of delivering quality care in light of an aging population.

One of the promises of mHealth is the development and promotion of mobile applications that support individuals in adopting a healthier lifestyle. As Neelie Kroes, the former vice-president of the EC, puts it: 'mHealth will reduce costly visits to hospitals, help citizens take charge of their own health and wellbeing, and move towards prevention rather than cure.' In other words, the EC envisions that mHealth empowers citizens to take control of their health by making physical processes relevant to one's health status that would otherwise remain invisible. It is thus expected that when citizens act upon this newly acquired knowledge, they are empowered to adopt a more healthy lifestyle accordingly. It is projected that this will lead to a decrease in hospital visits because so-called lifestyle related diseases (eg, obesity or diabetes type II) are more likely to be prevented.

The optimism of the EC is not universally shared. Some researchers worry that the promotion of using health monitoring technologies (HMAs) is exemplary of how medicine increasingly penetrates into our daily lives, thereby depriving citizens of the possibility to make autonomous choices with regard to their health. Some treat the potential decrease in au- 
tonomy as a practical concern that potentially hampers the successful implementation of mHealth. ${ }^{3}$ Others point to a deeper problem, namely that medical concerns (eg, contributing to the physical wellbeing of citizens) are deeply intertwined with political (eg, decrease in hospital costs) and commercial ones (eg, profit maximization of app developers), and that citizens are increasingly pushed to act upon norms that are unwarrantedly forced upon them. ${ }^{4} \mathrm{~A}$ central concern here is that mHealth is accompanied with unwarranted paternalism, which may make its introduction either practically difficult or ethically undesirable.

The growing ethical literature on mHealth tends to focus on how it increases or decreases the autonomy of users when making decisions related to their health status, but generally glosses over the question of how and if HMAs can establish a relationship between individuals and the 'healthiness' of their lifestyle. In this paper, this question will be explored by focusing on how one experiences and understands oneself as 'healthy' or as having a 'healthy lifestyle'. Only when this question is addressed can we start to appreciate how HMAs shape such experiences or understandings, such that it's influence on the extent to which citizens can make autonomous choices with regard to their health status can be analyzed.

Through a phenomenological analysis of health, I show that our 'health' or the 'healthiness' of our lifestyle is transparent to us (eg, is not something that can be explicitly related to). As a consequence, mHealth is - phenomenologically speaking — unable to make present 'health' or our 'healthy lifestyle' as a totality. I argue that this places limits on the extent to which it can be understood as something that (dis)empowers citizens to take responsibility for their own health. Rather, so I suggest, HMAs give rise to a specific relation with one's physical or biological body, instead of making present one's health status. Therefore, discussions about HMAs should not be exclusively focus on how they (dis)empower citizens, but additionally focus on evaluating how our physical body is drawn out of its transparency.

\section{Using mHealth to Promote a Healthy Lifestyle}

mHealth is an umbrella term covering (in the definition of the World Health Organization) 'medical and public health practices supported by mobile devices such as mobile phones, patient monitoring devices, personal digital assistants, and other wireless devices. ${ }^{5}$ Mobile applications that help citizens to adopt a (more) healthy lifestyle are deemed increasingly important in health promotion circles. Such apps allegedly help changing unhealthy habits by offering real-time feedback on the user's behavior by making them aware of 'unhealthy' habitual patterns and/or send motivational messages helping to change their behavior. Often, apps are developed to increase control on a specific kind of behavior, such as food intake, sleep, or physical activity. The promise of the widespread use of such apps is that they help prevent lifestyle related diseases, thereby contributing to the overall decrease of hospital treatments in the future.

A prominent example of a HMA is Fitbit, which can be connected to a smartwatch and allows the tracking of several aspects of one's daily activity, such as sleep, steps, or amount of burned calories. Another example is Habitica, an app that is promoted as helping users to set their own lifestyle goals and track their progress in realizing those (eg, by finishing a specific to-do list, going to the gym more often, having sufficient social interactions). The European Commission promotes the use of such apps as a form of 'digital empowerment,' because they allegedly help users to make informed decisions about their health status by making explicit the relation between habitual patterns and relevant health parameters. ${ }^{6}$

The long-term efficacy of HMAs is unclear. ${ }^{7}$ For example, a 2014 survey found that over a third of the users of commercially available trackers had stopped using them within 6 months. ${ }^{8}$ Despite such findings, it remains to be assumed that mHealth has a positive impact on adopting a healthy lifestyle, the only prob-

3 Jessica Morley and Luciano Floridi, 'The Limits of Empowerment: How to Reframe the Role of mHealth Tools in the Healthcare Ecosystem' (2019) Science and Engineering Ethics

4 Ignaas Devisch and Stijn Vanheule, 'Foucault at the Bedside: A Critical Analysis of Empowering a Healthy Lifestyle' (2015) 21 Journal of Evaluation in Clinical Practice 3, 427-432

5 World Health Organization, 'mHealth: New Horizons for Health Through Mobile Technologies' Global Observatory for eHealth Series (2011) 3, 6

$6 \quad(\mathrm{n} 1)$

7 Jing Zhao, Becky Freeman, and Mu Li, 'Can Mobile Phone Apps Influence People's Behavior Change? An Evidence Review' (2016) 18 Journal of Medical Internet Research 11, e287

8 Dan Ledger and Daniel McCaffrey, 'How the Science of Human Behavior Change Offers the Secret to Long-Term Engagement' (2014) 
lem remaining how to find a technical solution to the lack of long-term efficacy. Medical and commercial interests coincide here: app users supposedly benefit from the use of technologies helping them to adopt a more healthy lifestyle, and app developers by making profit when their apps are used more frequently. The latter aspect is also explicitly present in the vision of the EC, as evidenced by Neelie Kroes's statement that mHealth 'is also a great opportunity for the booming app economy and for entrepreneurs'. In this framing, there are seemingly only winners when the long-term efficacy of HMAs is ensured.

Many critical scholars do not share the optimism of the EC, and stress that we cannot assume beforehand the desirability of turning citizens into objects of surveillance and persuasion that are willing and able to act upon measurements and assessments by HMAs. ${ }^{10}$ A central criticism is the potential conflict between the idea that mHealth contributes to 'digital empowerment' and the political and commercial interests surrounding mHealth. In fact, so it is argued, HMAs do not help individuals to take responsibility for their own health, because a large amount of choices that were previously left to the individual are now outsourced to an external party that has a specific normative conception about what constitutes a healthy lifestyle strongly influenced by the quantitative model of the biomedical sciences. ${ }^{11} \mathrm{Al}-$ though qualitative studies on the Quantified Self movement showed that users integrate quantitative measurements of bodily parameters into broader personal narratives, ${ }^{12}$ the question remains whether or not HMAs make citizens increase the responsibility of their own health.

Additionally, app developers have an economic interest in persuading citizens to use HMAs, such that

$9 \quad($ n 2$)$

10 Deborah Lupton, 'M-Health and Health Promotion: The Digital Cyborg and Surveillance Society' (2012) 10 Social Theory \& Health 3, 229-244

11 (n 4)

12 Tamar Sharon, 'Self-Tracking for Health and the Quantified Self: Re-Articulating Autonomy, Solidarity, and Authenticity in an Age of Personalized Health Care' (2017) 30 Philosophy \& Technology 1, 93-121

13 Federica Luvicero and Barbara Prainsack, 'The Lifestylisation of Healthcare? 'Consumer Genomics' and Mobile Health as Technology for Healthy Lifestyle' (2015) 4 Applied \& Translational Genomics 1, 44-49

14 Havi Carel, Phenomenology of IIIness (Oxford University Press 2016) 1 health related concerns of citizens have become intertwined with the concern of companies to maximize financial profit. ${ }^{13}$ Because of this, it cannot be assumed that the development of HMAs that more effectively persuade citizens to remain using them primarily is driven by health-related interests only. Since companies benefit financially from the longterm use of HMAs, it seems that they would profit from users that do not make autonomous choices, but remain compliant with the specific conception of health embodied in a specific application. Such criticisms point to the fact that the EU slogan that mHealth leads to the 'digital empowerment' of citizens threatens to conceal hidden political and/or economic agendas.

Notwithstanding the relevance of such criticism, their focus on how they (dis)empower citizens to make responsible decisions about their own health bypasses the question to what extent HMAs can establish a relationship between individuals and their health. In the remainder of this paper, I use a phenomenological approach to address exactly this question by exploring whether and how measurements of patterns of bodily function and activity make present the 'healthiness' of one's lifestyle.

\section{Phenomenology and the Transparency of Health}

In this section, I draw on phenomenology to show that our own health is something that goes generally unnoticed (ie, health is transparent), as it is not directly present in our experience. Phenomenology is a branch of philosophy that studies how we experience and understand ourselves and the things around us from a first-person perspective. In the philosophy of medicine, there has been a growing interest in using phenomenology for studying experiences of health and illness that are easily neglected in the dominant biomedical perspective on the human body. ${ }^{14}$

One of the central distinctions in phenomenology is the one between our objective body and our lived body. The objective body refers to the body qua its biology, to an object in which physiological processes occur that can be analyzed objectively by the biomedical sciences; from this perspective the body is viewed as one of the objects encountered in the world. The lived body, on the contrary, refers to the body 'as 
[...] experienced by the person whose body it is..15 Approached in this way, our body is not an object amongst others but a medium through which we understand and experiences ourselves and our environment. Whereas the objective body can be analyzed from a third-person perspective, the lived body can only be experienced from a first-person perspective. $^{16}$

Havi Carel uses this distinction to criticize the biomedical sciences for viewing health and illness almost exclusively as physiological states in the objective body, thereby threatening to neglect that health and illness are primarily ways of experiencing the world through our lived body. What does this distinction reveal about our everyday experience of health?

According to Carel, we never experience 'health' directly. We experience the world around us through our body, making it such that our body is transparent. Although a body always is both a lived body and an objective body, in everyday healthy experience, these two are normally harmoniously aligned, such that we 'do not experience [our body] explicitly [...] or thematise it as an object of attention. ${ }^{17}$ For example, when eating I do not experience the bodily movements required to move food from my plate into my mouth, and neither do I explicitly thematise how my digestive system processes what I am eating. Instead, I am engaged in the activity of eating and immersed in the tasting of the food without paying attention to the bodily movements and processes involved in this activity.

Only when our digestive system prevents us from engaging in the activities that we like to pursue, our body becomes an object of attention. Yet, such cases in which the transparency of our body is disrupted do not necessarily direct our attention explicitly towards our health, because they still appear within a totality of bodily certainty. That is, 'we feel tacitly confident (or rather, we do not normally question) that our bodies will continue to function in a similar fashion in which they have in the past'. ${ }^{18}$ The habits that we have developed throughout our lives constitute a meaningful whole that is not directly experienced, yet implicitly offers a horizon against which the things we experience and the projects we engage in appear. For example, when I am suffering from a headache, this might prevent me from playing soccer today, but I expect that I will be able to do so the next week or the week after. Consequently, the world remains familiar to me because I tacitly assume that
I will be capable of executing such habits in the near future and expect that my body does not disrupt the routinely habitual way through which I understand myself and mine environment.

Carel suggests to make visible the ordinary experience of bodily certainty by looking at cases of its breakdown (ie, when it is disrupted by illness). Illness makes the familiar world of a person uncanny, thereby making it cease to be familiar, because the ill person is 'withdrawn from the world and focused on her body.' ${ }^{19}$ Illness gives rise to an experience of what Carel calls bodily doubt, which disrupts the meaningful whole we are immersed in ordinarily. It does so by destroying the experiences of continuity, transparency and trust: it becomes uncertain if it is possible to engage in the habitual actions needed to pursue our goals (continuity). Because of this the body becomes thematised as a problem and no longer taken-for-granted (transparency), which makes explicit that bodily certainty can no longer be upheld (trust). By explicitly thematising the body, illness transforms the world in which we live into a no longer familiar place. This experience calls for the ongoing evaluation of whether the actions we would like to undertake (and expect to be able to undertake) can be executed through our body.

This analysis point to the fact that our 'health' or the 'healthiness' of our lifestyle is a transparent meaningful totality allowing for the ongoing execution of our habits, instead of a measurable object. Following Carel, we can say that this meaningful totality only disappears from view in cases of bodily doubt. If this is correct, it places a limit on the extent to which HMAs, by providing knowledge of certain physiological parameters of the objective body, indeed allow for making informed decisions about our health. After all, if health is a meaningful horizon constituted by the possibility of the ongoing realization of habitual patterns, it evades explicit evaluation. The crucial question is, then, what still might be the contribution of HMAs for establishing lifestyle change in the light of this phenomenological analysis.

$\begin{array}{lr}15 & \text { ibid } 46 \\ 16 & \text { ibid } 26 \\ 17 & \text { ibid } 55 \\ 18 & \text { ibid } 89 \\ 19 & \text { ibid } 92\end{array}$ 


\section{A Healthy Lifestyle: More Than the Sum of Its Parts?}

Interestingly, the EC's narrative that HMAs will empower citizens to have increased control of their own health and the phenomenological perspective are grounded in a similar intuition: both conceive of health as something applying to the lifestyle of a person as a whole, instead of applying to isolated physiological processes. In this section, I use the distinction between objective body and lived body to make clear how these views diverge when it comes to how health as a whole must be understood.

HMAs allow for gathering increasingly precise information about the physiological status and habitual movement of the objective body. For example, a step counter gives exact information about how often we walk, or monitoring closely the food we consume precisely gives us exact information about our calorie intake. These apps, then, can send motivational messages in the case when we have not walked a certain distance yet, or consume ingredients that will likely lead to a weight increase. In the EU narrative, it seems to be stipulated that changes in one's physical activity and/or food intake will make citizens more healthy, because these allegedly will prevent the coming into existence of physiological processes that eventually give rises to certain diseases. The combination of acquiring knowledge of one's habitual patterns and the motivational nudges of HMAs, then, would give rise to increasingly precise information about one's health that can be subsequently be acted upon.

Through this focus on measuring the objective body, it seems to be assumed that the totality of one's health is something that citizens cannot relate to yet, and HMAs are thought to contribute to establishing this relation in the future by explicating previously invisible habitual patterns. An increasingly large set of habitual and/or physiological patterns revealed then, so it seems to be assumed, will cumulatively generate a total image of the 'healthiness' of one's lifestyle, such that a relationship with one's health can be established.

From a phenomenological perspective, our habits are not primarily to be understood as measurable activities, but rather as constitutive of a totality in which

20 ibid 89 we can act in the environment in an unproblematic and meaningful way. When analyzing how the world appears to us through our lived body, phenomenologists showed that the execution of habits is mostly done unreflectively and more or less effortlessly, such that we do not direct our experience towards the body that allows for the execution of habitual actions. An implicit sense of bodily certainty make it such that we expect that we remain capable to engage in executing our habitual patterns. As a consequence, 'bodily certainty $[\ldots]$ is a necessary constituent of existential feelings that makes its bearer present in a world by offering her a meaningful horizon in which things and projects can appear. ${ }^{20}$ Phenomenologically speaking, the healthiness of one's lifestyle is thus not to be understood as the aggregate sum of a set of habitual activities, but instead as the appearance of a meaningful horizon against which the projects we intend to engage in appear as meaningful.

If this is correct, then the use and promotion of HMAs cannot be said to offer citizens objective knowledge about the healthiness of one's lifestyle, as health is something irreducible to measurements of the objective body. As the horizon against which our habits are effortlessly executed, health remains transparent for the individual. In light of this analysis of health, it becomes clear that the EU vision stating that HMAs will help individuals to take control of their own health problematically reduces health to a set of measurable properties of the objective body. As shown in this section, this perspective neglects that health — as a totality—cannot be a direct object of experience, but is something through which we experience and understand the world around us.

\section{Conclusion: HMAs as Enabling a Relation Between Objective and Lived Body}

Even though that HMAs are no solution to the transparency of health, it should not be forgotten that they might reveal relevant information to users. But what constitutes the relevancy of this information?

Let us first highlight something that my phenomenological analysis of health has thus far not explicitly accounted for: the fact that HMAs do measure certain habitual patterns of the objective body previously invisible to their users. Indeed, HMAs do specifically thematise certain aspects of the objective body, 
such that these are turned into potential objects of experience. For example, when monitoring food intake, the amount of calories that we consume becomes present as a thing in the world with which a relationship can be established. As a consequence, we are put into a position to take a critical stance towards whether or not this amount of calories correlates with our goals (eg, being able to swim with my child during the weekend). In other words, HMAs have the potential to draw habits out of their transparency by turning them into an explicit object of attention.

Temporarily at least, HMAs turn our body into a central aspect of our world, thereby disrupting the harmony between objective body and lived body. This is not to say that HMAs question the desirability of a specific lifestyle altogether. Havi Carel reserves such disruptions for cases of bodily doubt in which the meaningful horizon against which our habits makes sense ceases to exist. ${ }^{21}$ HMAs draw the body out of its transparency less dramatically, but nevertheless help establish a relationship between lived body and objective body, which invites questions of how habitual actions link to the projects we like to pursue.
The relationship between objective and lived body can take different forms. In a relationship of control, measurements of the objective body dictate the kind of habits that one should pursue with reference to a certain conception of healthiness. An example of this would be an app that uses a certain amount of daily calorie intake (say 2000 calories) as a norm of health to which one should conform. In such a case, it is enforced what is a good relationship between lived and objective body; a relationship that conforms to the health norm being set based on certain characteristics of the objective body. A relationship of play-one that does more justice to the phenomenological perspective on health developed in this paper-would be one that invite users to link their habitual patterns to their own projects and goals, without enforcing an external health norm. From this perspective, the central benefit of HMAs would be that a relationship between objective and lived body is established, leaving it open to users to set how this new relationship should be formative of their projects.

21 (n 18) 